\title{
Elevated peripheral blood lymphocyte- to-monocyte ratio predicts a favorable prognosis in the patients with metastatic nasopharyngeal carcinoma
}

Rou Jiang ${ }^{1,2,3+}$, Xiu-Yu Cai ${ }^{1,4+}$, Zhong-Han Yang ${ }^{5+}$, Yue Yan ${ }^{1,3}$, Xiong Zou ${ }^{1,2}$, Ling Guo ${ }^{1,2}$, Rui Sun ${ }^{1,2}$,

Dong-Hua Luo ${ }^{1,2}$, Qiu-Yan Chen ${ }^{1,2}$, Pei-Yu Huang ${ }^{1,2}$, Yan-Qun Xiang ${ }^{1,2}$, Xing Lu ${ }^{1,2}$, Lin Wang ${ }^{1,2}$, Wei-Xiong Xia ${ }^{1,2}$,

Hai-Qiang Mai ${ }^{1,2}$ and Ming-Yuan Chen ${ }^{1,2^{*}}$

\begin{abstract}
Introduction: Patients with metastatic nasopharyngeal carcinoma (NPC) have variable survival outcomes. We have previously shown that an elevated peripheral blood lymphocyte-to-monocyte ratio (LMR) is associated with an increased metastatic risk in patients with primary NPC. The present study aimed to investigate the prognostic value of pretreatment LMR in a large cohort of metastatic NPC patients.

Methods: Clinical data of 672 patients with metastatic NPC diagnosed between January 2003 and December 2009 were analyzed. The peripheral lymphocyte and monocyte counts were retrieved, and LMR was calculated. Receiver operating characteristic (ROC) curve analysis and univariate and multivariate COX proportional hazards analyses were performed to evaluate the association of LMR with overall survival (OS).

Results: Univariate analysis revealed that an elevated absolute lymphocyte count $\left(\geq 1.390 \times 10^{9} / \mathrm{L}\right)$ and LMR $(\geq 2.475)$ as well as a decreased monocyte count $\left(<0.665 \times 10^{9} / \mathrm{L}\right)$ were significantly associated with prolonged OS. Multivariate Cox proportional hazard analysis showed that LMR (hazard ratio [HR] $=0.50,95 \%$ confidence interval $[\mathrm{Cl}]=0.41-0.60, P<0.001)$, absolute lymphocyte count $(\mathrm{HR}=0.77,95 \% \mathrm{Cl}=0.64-0.93, P=0.007)$, and monocyte count $(H R=1.98,95 \% \mathrm{Cl}=1.63-2.41, P<0.001)$ were independent prognostic factors. By stratification analyses, only LMR remained a significant predictor of prognosis.
\end{abstract}

Conclusion: We identified pretreatment LMR as an independent prognostic factor for patients with metastatic NPC. Independent validation of our findings is needed.

Keywords: Metastatic nasopharyngeal carcinoma, Lymphocyte count, Monocyte count, Lymphocyte-to-monocyte ratio, Overall survival, Prognosis

\section{Background}

Nasopharyngeal carcinoma (NPC) is a squamous cell carcinoma that occurs in the epithelial lining of the nasopharynx, with high incidence recorded in South

\footnotetext{
* Correspondence: chmingy@mail.sysu.edu.cn

${ }^{\dagger}$ Equal contributors

'Sun Yat-sen University Cancer Center; State Key Laboratory of Oncology in South China; Collaborative Innovation Center for Cancer Medicine, Guangzhou, Guangdong 510060, P. R. China

${ }^{2}$ Department of Nasopharyngeal Carcinoma, Sun Yat-sen University Cancer Center, Guangzhou, Guangdong 510060, P. R. China

Full list of author information is available at the end of the article
}

China and Southeast Asia [1, 2]. With the increasing application of high-precision radiotherapy, distant failure is expected to become a predominant cause of death from NPC $[3,4]$. Once metastasis is diagnosed, the overall survival (OS) of patients is typically under 15 months with palliative chemotherapy. Nevertheless, retrospective studies have shown great differences in the survival outcomes in patients with variable affected anatomic sites and different numbers of metastases $[5,6]$. For specific subgroups, OS may exceed 10 years [6]. Therefore, a valuable marker to predict prognosis is desirable to 
facilitate individualized treatments and thus to achieve better outcomes for patients with metastatic NPC.

In the last decade, pretreatment peripheral differential leukocytes, such as lymphocytes and monocytes, have been found to be associated with prognosis in various cancers. A high pretreatment lymphocyte count has been determined to be associated with the good prognosis of patients with acute lymphoblastic leukemia [7], metastatic gastric cancer [8], and NPC [9]. A high monocyte count has been found to be a poor independent prognostic factor in patients with diffuse large B-cell lymphoma and metastatic melanoma [10]. An elevated lymphocyte-to-monocyte ratio (LMR) has been reported to be a prognostic factor for clinical outcome in patients with diffuse large Bcell lymphoma and Hodgkin's lymphoma [11]. Recently, we have shown that an elevated LMR is associated with an increased metastatic risk in patients with primary NPC [12]. However, there have been few studies of the prognostic value of LMR in patients with metastatic NPC. Therefore, the current study was designed to analyze the effect of pretreatment LMR on OS in these patients.

\section{Patients and Methods}

\section{Patient selection and data collection}

Clinical data of patients with metastatic NPC referred to Sun Yat-sen University Cancer Center (SYSUCC) between January 2003 and December 2009 were reviewed. All of the included patients met the following criteria: 1) pathologically confirmed World Health Organization (WHO) type II or III NPC; 2) radiographically detectable metastatic disease; 3) a Karnofsky Performance Status score of $\geq 70$; and 4) available clinical information and laboratory data at the diagnosis of metastasis. The exclusion criteria were as follows: 1) patients with a self-reported acute infection or hematologic disorder and 2) those with another type of malignancy. The Union for International Cancer Control/American Joint Committee on Cancer (UICC/AJCC) TNM classification system (6th edition, 2002) was used for staging. This study protocol was approved by the Clinical Ethics Review Board of SYSUCC.

As part of the physical examination, peripheral blood was collected before treatment, and both peripheral lymphocytes and monocytes were counted using a Sysmex XE-5000 automated hematology analyzer (Sysmex, Kobe, Japan). The peripheral LMR was calculated as the ratio of the absolute peripheral lymphocyte count to monocyte count. The serum antibody titers of Epstein-Barr virus (EBV) immunoglobulin A against virus capsid antigen (VCA/IgA) and early antigen
(EA/IgA) were detected by enzyme-linked immunosorbent assay [13].

\section{Treatment and follow-up}

According to our institutional guidelines for the palliative treatment of metastatic NPC, cisplatin-based systemic chemotherapy was provided to all patients as a basic treatment. Definitive radiotherapy targeting both the primary tumor and its regional lymph nodes (locoregional radiotherapy, lrRT) was administered to the patients with metastasis at presentation for local symptomatic relief or as a part of a multidisciplinary approach, as previously described [14-16]. The evaluation of tumor response to therapy was based on a computed tomography (CT) or magnetic resonance imaging (MRI) scan. After the treatment was completed, the patients were evaluated at 3-month intervals for the first 3 years and every 6 months thereafter or until death. The last follow-up date was December 31, 2013 for all available patients.

\section{Statistical analysis}

Statistical analyses were performed using SPSS software (version 16.0, SPSS Inc., Chicago, IL, USA). OS was defined as the period between the first diagnosis of metastatic NPC and death or the last follow-up. The receiver operating characteristic (ROC) curve analysis was performed to select the most appropriate cut-off points for absolute lymphocyte and monocyte counts as well as LMR to stratify the patients at high risk of malignancy-related death. Univariate and multivariate analyses of clinicopathologic variables were performed using Cox proportional hazards regression models. Actuarial OS was plotted against time using the Kaplan-Meier method, and differences between the survival curves were assessed using the log-rank test. The correlation of LMR with different clinicopathologic characteristics was evaluated by Spearman's rank correlation coefficient $(r)$. The chi-square test was used to analyze differences in proportions. A two-sided $P<0.05$ was considered significant.

\section{Results}

\section{Patients' characteristics}

In total, the data for 672 patients were retrievable. The baseline patient characteristics are shown in Table 1. The median age at the time of diagnosis was 46 years (range, 13-79 years), and 546 (81.2\%) patients were male. Two hundred and ninety-seven (44.2\%) patients had distant metastases at presentation. The bones (58.3\%) were the most common site of metastasis, followed by the liver (34.1\%), lung (31.2\%), and extraregional lymph nodes (26.0\%). Most of the patients (84.4\%) had metastases at 
Table 1 Clinicopathologic characteristics of the 672 patients with metastatic nasopharyngeal carcinoma (NPC)

\begin{tabular}{|c|c|c|c|c|}
\hline Characteristic & Total (cases) & Deaths [cases (\%)] & Unadjusted HR (95% Cl) & $P$ value \\
\hline Total & 672 & 458 & & \\
\hline \multicolumn{5}{|l|}{ Gender } \\
\hline Male & 546 & $371(67.9)$ & Reference & \multirow[t]{2}{*}{0.700} \\
\hline Female & 126 & $87(69.0)$ & $1.05(0.83,1.32)$ & \\
\hline \multicolumn{5}{|l|}{ Age (years) } \\
\hline$<46$ & 316 & $213(67.4)$ & Reference & \multirow[t]{2}{*}{0.124} \\
\hline$\geq 46$ & 356 & $245(68.8)$ & $1.16(0.96,1.39)$ & \\
\hline \multicolumn{5}{|l|}{ T stage* } \\
\hline $\mathrm{T} 1-2$ & 273 & $184(67.4)$ & Reference & \multirow[t]{2}{*}{0.975} \\
\hline T3-4 & 399 & $274(68.7)$ & $1.00(0.83,1.20)$ & \\
\hline \multicolumn{5}{|l|}{ N stage* } \\
\hline No-1 & 273 & $172(63.0)$ & Reference & \multirow[t]{2}{*}{$<0.001$} \\
\hline $\mathrm{N} 2-3$ & 399 & $286(71.7)$ & $1.42(1.18,1.72)$ & \\
\hline \multicolumn{5}{|c|}{ Metastasis at presentation } \\
\hline Absent & 375 & $261(69.6)$ & Reference & \multirow[t]{2}{*}{0.825} \\
\hline Present & 297 & $197(66.3)$ & $0.98(0.81,1.18)$ & \\
\hline \multicolumn{5}{|c|}{ Number of metastatic lesions } \\
\hline 1 & 105 & $51(48.6)$ & Reference & \multirow[t]{2}{*}{$<0.001$} \\
\hline$\geq 2$ & 567 & $407(71.8)$ & $2.06(1.54,2.77)$ & \\
\hline \multicolumn{5}{|c|}{ Bone metastasis } \\
\hline Absent & 280 & $196(70.0)$ & Reference & \multirow[t]{2}{*}{0.862} \\
\hline Present & 392 & $262(66.8)$ & $1.02(0.85,1.22)$ & \\
\hline \multicolumn{5}{|c|}{ Liver metastasis } \\
\hline Absent & 443 & $283(63.9)$ & Reference & \multirow[t]{2}{*}{$<0.001$} \\
\hline Present & 229 & $175(76.4)$ & $1.62(1.34,1,95)$ & \\
\hline \multicolumn{5}{|c|}{ Lung metastasis } \\
\hline Absent & 462 & $309(66.9)$ & Reference & \multirow[t]{2}{*}{0.847} \\
\hline Present & 210 & $149(71.0)$ & $0.98(0.81,1.19)$ & \\
\hline \multicolumn{5}{|c|}{ Extraregional lymph node metastasis } \\
\hline Absent & 497 & $328(66.0)$ & Reference & \multirow[t]{2}{*}{0.060} \\
\hline Present & 175 & $130(74.3)$ & $1.22(0.99,1.49)$ & \\
\hline \multicolumn{5}{|l|}{ EBV VCA/lgA } \\
\hline$<1: 80$ & 80 & $48(60.0)$ & Reference & \multirow[t]{3}{*}{0.413} \\
\hline $1: 80-1: 320$ & 395 & $270(68.4)$ & $1.15(0.84,1.56)$ & \\
\hline$\geq 1: 640$ & 197 & $140(71.1)$ & $1.17(0.85,1.63)$ & \\
\hline \multicolumn{5}{|l|}{ EBV EA/lgA } \\
\hline$<1: 10$ & 128 & $84(65.6)$ & Reference & \multirow[t]{3}{*}{0.385} \\
\hline 1:10-1:20 & 216 & $149(69.0)$ & $0.97(0.74,1.27)$ & \\
\hline$\geq 1: 40$ & 328 & $225(68.6)$ & $0.91(0.71,1.16)$ & \\
\hline \multicolumn{5}{|c|}{ Lymphocyte count $\left(\times 10^{9} / \mathrm{L}\right)$} \\
\hline$<1.390$ & 340 & $250(73.5)$ & Reference & 0.002 \\
\hline$\geq 1.390$ & 332 & $208(62.7)$ & $0.75(0.63,0.90)$ & \\
\hline
\end{tabular}


Table 1 Clinicopathologic characteristics of the 672 patients with metastatic nasopharyngeal carcinoma (NPC) (Continued)

\begin{tabular}{lccl}
\hline Characteristic & Total (cases) & Deaths [cases (\%)] & Unadjusted HR $(95 \% \mathrm{Cl})$ \\
\hline $\begin{array}{l}\text { Monocyte count }\left(\times 10^{9} / \mathrm{L}\right) \\
<0.665\end{array}$ & 428 & $263(61.4)$ & Reference \\
$\geq 0.665$ & 244 & $195(80.0)$ & $2.17(1.80,2.62)$ \\
LMR & & & \\
$<2.475$ & 335 & $269(80.3)$ & Reference \\
$\geq 2.475$ & 337 & $189(56.1)$ & $0.45(0.37,0.54)$ \\
\hline
\end{tabular}

$\mathrm{HR}$, hazard ratio; $\mathrm{Cl}$, confidence interval; EBV, Epstein-Barr virus; VCA/lgA, immunoglobulin $\mathrm{A}$ against viral capsid antigen; EA/lgA, immunoglobulin $\mathrm{A}$ against early antigen; LMR, lymphocyte-to-monocyte ratio. ${ }^{*}$ The American Joint Committee on Cancer (AJCC) 2002 system was used for staging at the diagnosis of NPC

multiple sites at the time of diagnosis. By the last follow-up, $458(68.2 \%)$ of the 672 patients died. The 1-, 2-, and 3-year OS rates for the entire patient cohort were $81.1 \%, 52.5 \%$, and $37.2 \%$, respectively.

The mean lymphocyte and monocyte counts were $1.462 \times 10^{9} / \mathrm{L}$ (range, $0.100-5.850 \times 10^{9} / \mathrm{L}$ ) and $0.607 \times 10^{9} /$ L (range, $0.020-2.300 \times 10^{9} / \mathrm{L}$ ), respectively. The mean LMR was 3.219 (range, 0.180-69.000). The cut-off points of the absolute lymphocyte count, monocyte count, and LMR for OS as determined by ROC curve analyses were $1.390 \times 10^{9}$ / L, $0.665 \times 10^{9} / \mathrm{L}$, and 2.475 , respectively (Fig. 1 ). A low LMR $(<2.475)$ was significantly correlated with metastasis after radical therapy $(r=0.306, P<0.001)$, involvement of more than one metastatic site $(r=-0.150, P<0.001)$, liver metastasis $(r=-0.150, P<0.001)$, and extraregional lymph node metastasis $(r=-0.100, P=0.009)$ (Table 2).

\section{Univariate Cox proportional hazards regression analysis of clinicopathologic characteristics}

As shown in Table 1, the absolute lymphocyte count, monocyte count, LMR, and other clinicopathologic characteristics (including age, gender, $\mathrm{T}$ stage, $\mathrm{N}$ stage, metastasis at presentation, number of metastatic lesions, metastasis sites [i.e., the bones, liver, lungs, and extraregional lymph nodes], and EBV-related antibodies [i.e., serum VCA/IgA and IgA/EA antibodies]) were subjected to univariate analysis. A higher absolute lymphocyte count, lower absolute monocyte count, and higher LMR were associated with longer OS (Fig. 2). Other variables, including $\mathrm{N}$ stage (hazard ratio $[\mathrm{HR}]=$ 1.42, $95 \%$ confidence interval $[\mathrm{CI}]=1.18-1.72, P<0.001)$, number of metastatic lesions $(\mathrm{HR}=2.06,95 \% \mathrm{CI}=1.54$ 2.77, $P<0.001)$, and liver metastasis $(\mathrm{HR}=1.62$, $95 \%$ $\mathrm{CI}=1.34-1.95, P<0.001)$, were also found to be associated with OS (Table 1).

\section{Multivariate Cox proportional hazards regression analysis of clinicopathologic characteristics}

We used a multivariate model to adjust for the confounders of the association of LMR with survival. The results showed that a high LMR was an independent predictor of a favorable $\mathrm{OS}(\mathrm{HR}=0.50,95 \% \mathrm{CI}=$ 0.41-0.60, $P<0.001$ ) (Table 3 Model 1 , including LMR as a variable). In addition, both absolute
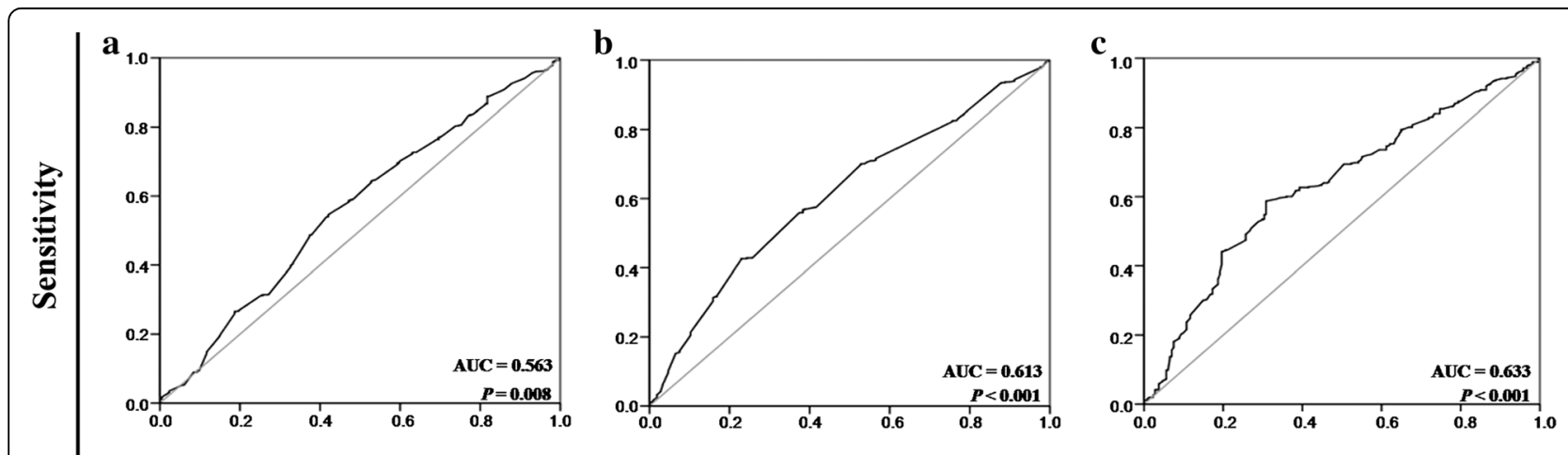

\section{1-Specificity}

Fig. 1 Receiver operating characteristic (ROC) curve analyses of cut-off points of baseline absolute lymphocyte and monocyte counts and lymphocyte-to-monocyte ratio (LMR) for overall survival (OS) analyses among patients with metastatic nasopharyngeal carcinoma (NPC). The cut-off points of the lymphocyte count (a), monocyte count (b), and LMR (c) for OS analyses were $1.390 \times 10^{9} / \mathrm{L}, 0.665 \times 10^{9} / \mathrm{L}$, and 2.475 , respectively. AUC, area under the ROC curve 
Table 2 Correlation of baseline LMR with clinicopathologic characteristics of patients with metastatic NPC

\begin{tabular}{|c|c|c|c|c|}
\hline Characteristic & $L M R<2.475$ & $L M R \geq 2.475$ & $r$ value & $P$ value \\
\hline Total & 335 & 337 & & \\
\hline \multicolumn{5}{|l|}{ Gender } \\
\hline Male & 277 & 269 & 0.037 & 0.342 \\
\hline Female & 58 & 68 & & \\
\hline \multicolumn{5}{|l|}{ Age (years) } \\
\hline$<46$ & 157 & 159 & -0.003 & 0.935 \\
\hline$\geq 46$ & 178 & 178 & & \\
\hline \multicolumn{5}{|l|}{ T stage } \\
\hline $\mathrm{T} 1-\mathrm{T} 2$ & 136 & 137 & $<0.001$ & 0.988 \\
\hline T3-T4 & 199 & 200 & & \\
\hline \multicolumn{5}{|l|}{ N stage } \\
\hline NO-N1 & 138 & 135 & 0.012 & 0.765 \\
\hline N2-N3 & 197 & 202 & & \\
\hline \multicolumn{5}{|c|}{ Metastasis at presentation } \\
\hline Absent & 238 & 137 & 0.306 & $<0.001$ \\
\hline Present & 97 & 200 & & \\
\hline \multicolumn{5}{|c|}{ Number of metastatic lesions } \\
\hline 1 & 34 & 71 & -0.150 & $<0.001$ \\
\hline$\geq 2$ & 301 & 266 & & \\
\hline \multicolumn{5}{|c|}{ Bone metastasis } \\
\hline Absent & 139 & 141 & -0.004 & 0.927 \\
\hline Present & 196 & 196 & & \\
\hline \multicolumn{5}{|c|}{ Liver metastasis } \\
\hline Absent & 197 & 246 & -0.150 & $<0.001$ \\
\hline Present & 138 & 91 & & \\
\hline \multicolumn{5}{|c|}{ Lung metastasis } \\
\hline Absent & 224 & 238 & -0.041 & 0.294 \\
\hline Present & 111 & 99 & & \\
\hline \multicolumn{5}{|c|}{ Extraregional lymph node metastasis } \\
\hline Absent & 233 & 264 & -0.100 & 0.009 \\
\hline Present & 102 & 73 & & \\
\hline \multicolumn{5}{|l|}{ EBV VCA/lgA } \\
\hline$<1: 80$ & 36 & 44 & -0.037 & 0.339 \\
\hline $1: 80-1: 320$ & 197 & 198 & & \\
\hline$\geq 1: 640$ & 102 & 95 & & \\
\hline \multicolumn{5}{|l|}{ EBV EA/lgA } \\
\hline$<1: 10$ & 56 & 72 & -0.055 & 0.151 \\
\hline $1: 10-1: 20$ & 109 & 107 & & \\
\hline$\geq 1: 40$ & 170 & 158 & & \\
\hline
\end{tabular}

Footnotes as in Table 1 


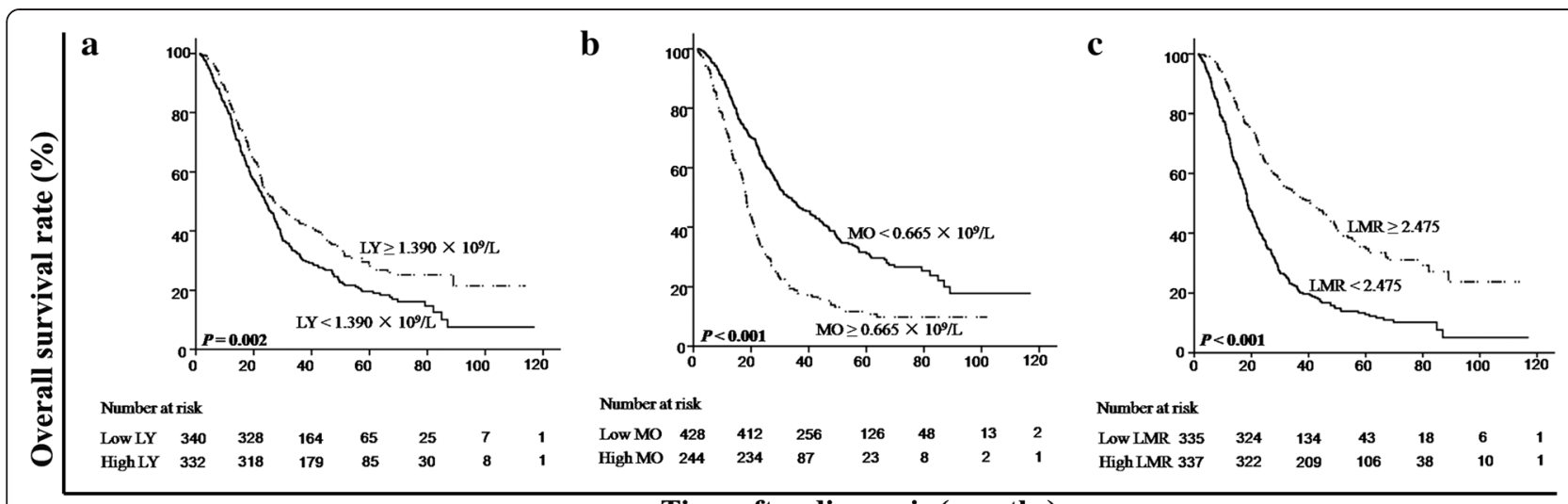

Time after diagnosis (months)

Fig. 2 Kaplan-Meier overall survival (OS) analysis for patients with metastatic NPC. a, the OS rate was higher in the patients with a high absolute lymphocyte count than in those with a low count $(P=0.002)$. $\mathbf{b}$, the OS rate was lower in the patients with a high absolute monocyte count than in those with a low count $(P<0.001)$. $\mathbf{c}$, the OS rate was higher in the patients with a high LMR than in those with a low LMR $(P<0.001)$. LY, lymphocyte; $\mathrm{MO}$, monocyte

lymphocyte and monocyte counts were analyzed for their independence from other covariates using the Cox model (Table 3 Model 2, including lymphocyte and monocyte counts as variables). LMR was not included here, considering the multicollinearity between LMR and absolute lymphocyte and monocyte counts. The results showed that the absolute lymphocyte count was an independent factor for a favorable prognosis $(\mathrm{HR}=0.77,95 \% \mathrm{CI}=0.64-0.93, P=0.007)$, whereas the absolute monocyte count was an independent inferior prognostic factor for patients with metastatic NPC $(\mathrm{HR}=1.98,95 \% \mathrm{CI}=1.63-2.41, P$ $<0.001$ ) (Table 3 Model 2). After stratification by $\mathrm{T}$ stage, $\mathrm{N}$ stage, metastasis at presentation, metastasis after radical therapy, number of metastatic lesions, and metastatic sites, only LMR remained a significant predictor of prognosis (Figs. 3, 4 and 5). Moreover, an advanced $\mathrm{N}$ stage, the presence of two or more lesions, and liver metastasis were shown to be independent indicators of short OS (Table 3).

\section{Discussion}

In the current study, we demonstrated that an elevated LMR was significantly associated with prolonged OS and was independent of the other variables assessed in predicting the prognosis of patients with metastatic NPC. Moreover, after stratification by $\mathrm{T}$ stage, $\mathrm{N}$ stage, metastasis at presentation, metastasis after radical therapy, number of metastatic lesions, and metastatic sites, LMR remained a significant predictor of prognosis.

There is substantial evidence in advanced cancer that the host systemic immune response is an important independent predictor of outcome and that pre-treatment measures of the systemic inflammatory immune response can be used to independently predict cancer patients' survival [17]. Among many systemic inflammatory measures, the white blood cell (WBC) subset count (the neutrophil count [18] or the neutrophil-to-lymphocyte ratio [19]) is well known as an independent prognostic factor for survival [17]. However, evidence that LMR may have a prognostic role in cancer is limited. Recent

Table 3 Multivariate analysis of prognostic factors in patients with metastatic NPC

\begin{tabular}{|c|c|c|c|c|}
\hline \multirow[t]{2}{*}{ Characteristic } & \multicolumn{2}{|c|}{ Model 1} & \multicolumn{2}{|c|}{ Model 2} \\
\hline & $\mathrm{HR}(95 \% \mathrm{Cl})$ & $P$ value & $\mathrm{HR}(95 \% \mathrm{Cl})$ & $P$ value \\
\hline N stage (N2-3 vs. N0-1) & $1.42(1.18,1.72)$ & $<0.001$ & $1.39(1.15,1.68)$ & 0.001 \\
\hline Number of metastatic lesions ( $\geq 2$ vs. 1 ) & $1.73(1.29,2.32)$ & $<0.001$ & $1.87(1.40,2.52)$ & $<0.001$ \\
\hline Liver metastasis (present vs. absent) & $1.38(1.14,1.67)$ & 0.001 & $1.29(1.06,1.57)$ & 0.010 \\
\hline Lymphocyte count $\left(\geq 1.390 \times 10^{9} / \mathrm{L}\right.$ vs. $\left.<1.390 \times 10^{9} / \mathrm{L}\right)$ & NA & NA & $0.77(0.64,0.93)$ & 0.007 \\
\hline Monocyte count $\left(\geq 0.665 \times 10^{9} / \mathrm{L}\right.$ vs. $\left.<0.665 \times 10^{9} / \mathrm{L}\right)$ & NA & NA & $1.98(1.63,2.41)$ & $<0.001$ \\
\hline LMR $(\geq 2.475$ vs. $<2.475)$ & $0.50(0.41,0.60)$ & $<0.001$ & NA & NA \\
\hline
\end{tabular}

NA, not applicable. Other footnotes as in Table 1 


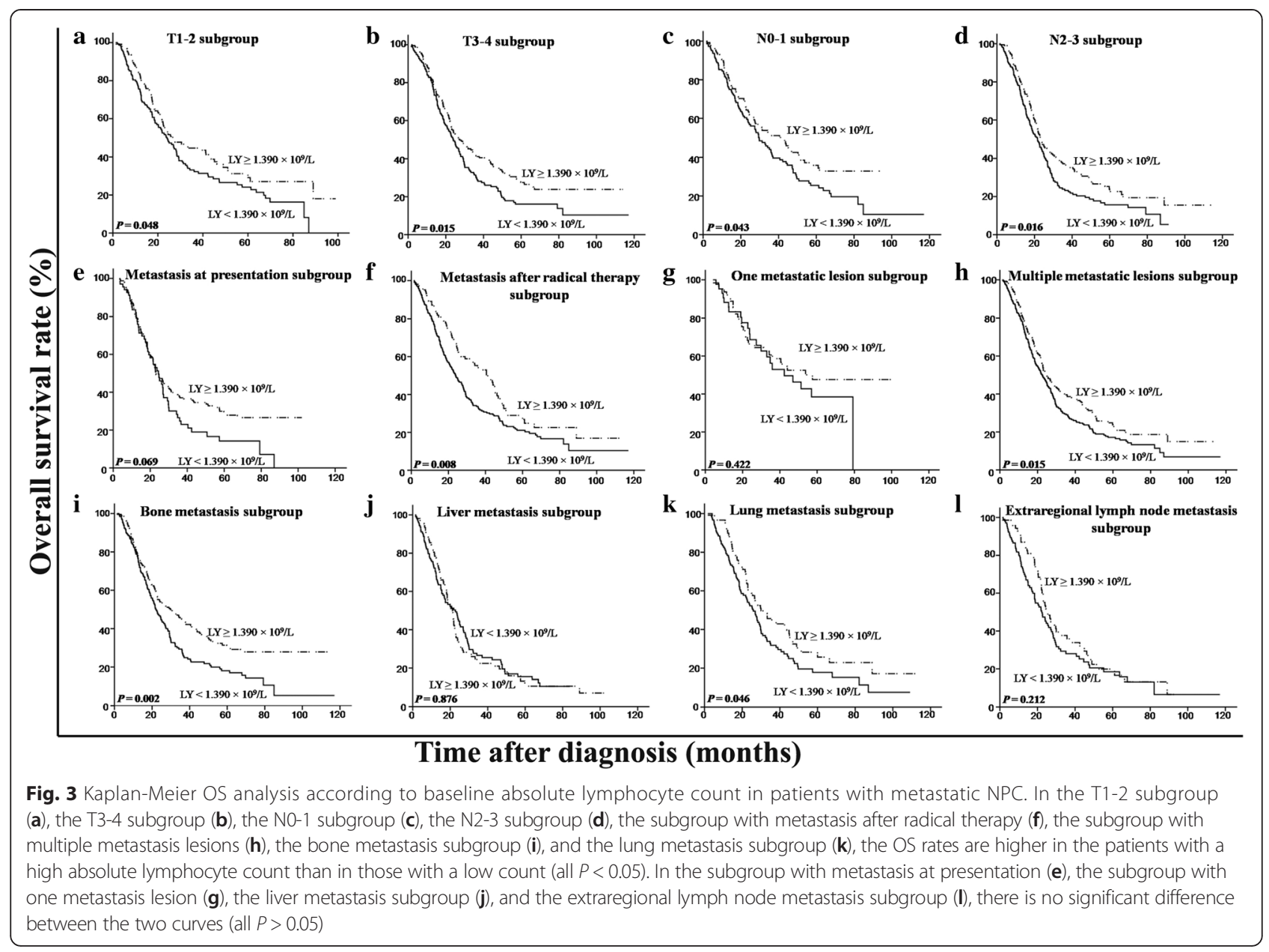

reports have indicated that LMR was positively associated with survival outcomes in classical Hodgkin's lymphoma [20], diffuse large B-cell lymphoma [21], metastatic non-small cell lung cancer [22], and NPC [12]. In the present study, we evaluated LMR as a prognostic indicator in 672 patients with metastatic NPC. Some of our results were consistent with previous findings. We found that an elevated LMR not only had a strong correlation with longer survival but also was an independent prognostic factor for survival, as determined by multivariate analysis using the Cox model. However, some of our results differed from those reported by Jin et al. [23], which have shown that the absolute lymphocyte count was not correlated with OS. In the current study, after adjusting for confounders, the absolute lymphocyte count remained as an independent prognostic factor for OS. The discordance between these two studies may be partially due to the different sample sizes: 672 patients were recruited in this study compared with 229 in the study by Jin et al. [23].
The mechanisms underlying the relationship between LMR and the prognosis of cancer patients remain unclear, which may be partially explained by the link between chronic inflammation and cancers [24-26]. It is a consensus that the adaptive immune system carries out immune surveillance and can eliminate newly formed tumors; however, effective adaptive immune responses are always suppressed in established tumors through several pathways, including the inhibition of dendritic cell differentiation and the activation and infiltration of regulatory $\mathrm{T}$ cells and tumor-associated macrophages [24]. Lymphocytes are crucial components of the adaptive immune system, and the presence of tumor-infiltrating lymphocytes has been reported to indicate the generation of an effective antitumor cellular immune response [27]. The peritumoral inflammatory response is thought to reflect the interaction between the tumor and the host. In previous studies, a high lymphocytic infiltrate has been linked with prolonged survival, independent of clinicopathologic characteristics, in breast cancer patients [28]. However, data supporting the association between intratumoral immune cells and blood-based 


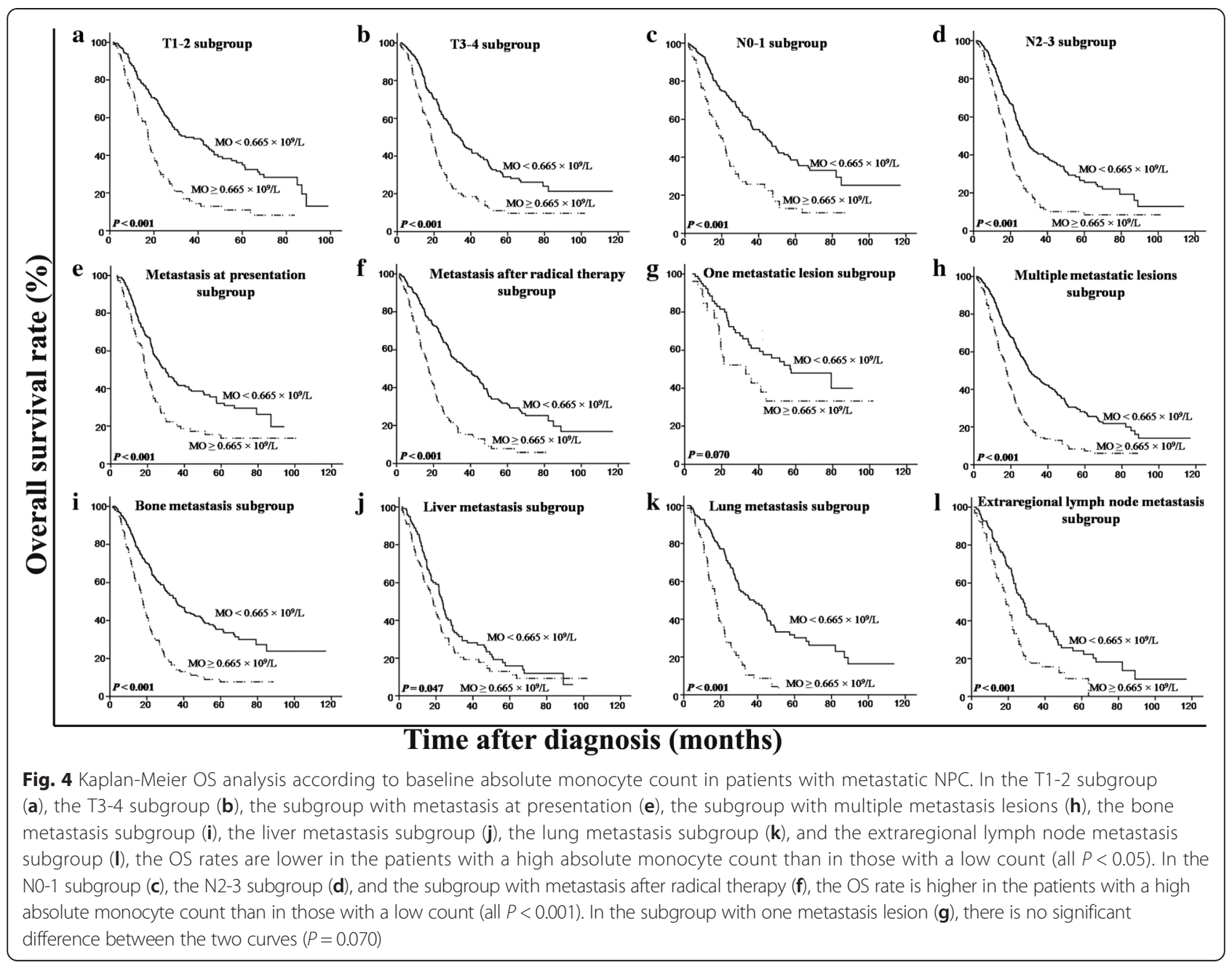

cells constituting the systemic inflammatory response with OS are sparse. Previous studies have demonstrated an association between a low peripheral blood lymphocyte count and short survival in patients with different types of cancer $[29,30]$. We have previously shown prolonged survival of primary NPC patients with elevated lymphocyte counts compared with those with decreased lymphocyte counts [12]. Monocytes are a subset of circulating white blood cells that can further differentiate into a range of tissue macrophages and dendritic cells [31]. It has been reported that monocytes secrete various proinflammatory cytokines, such as interleukin (IL)-1, IL-6, IL-10, and tumor necrosis factor- $\alpha$ (TNF- $\alpha$ ), which have been associated with short survival and poor prognosis in patients with malignancy [32, 33]. Moreover, monocytes release monocyte chemo-attractant protein-1 (MCP-1) upon stimulation and mediate tumor-associated macrophage infiltration in solid tumors, which have been shown to produce a variety of chemokines, such as transforming growth factor- $\alpha$ (TGF- $\alpha$ ), TNF- $\alpha$, IL-1, and IL-6, to promote tumorigenesis, angiogenesis, and distant metastasis of malignant tumors [34, 35]. As a consequence, a high absolute monocyte count may indicate poor prognosis. Our findings also showed that a high monocyte count was significantly associated with short survival in patients with metastatic NPC.

LMR, which is defined as the absolute lymphocyte count divided by the absolute monocyte count, may reflect the diverse effects of monocytes and lymphocytes on tumor progression. Previous studies have demonstrated that normal human monocytes suppress either the phytohemagglutinin- or antigen-induced lymphocyte proliferative response when the monocyteto-lymphocyte ratio is increased [36]. In the current study, although the lymphocyte count or monocyte count alone could predict the survival outcomes in 


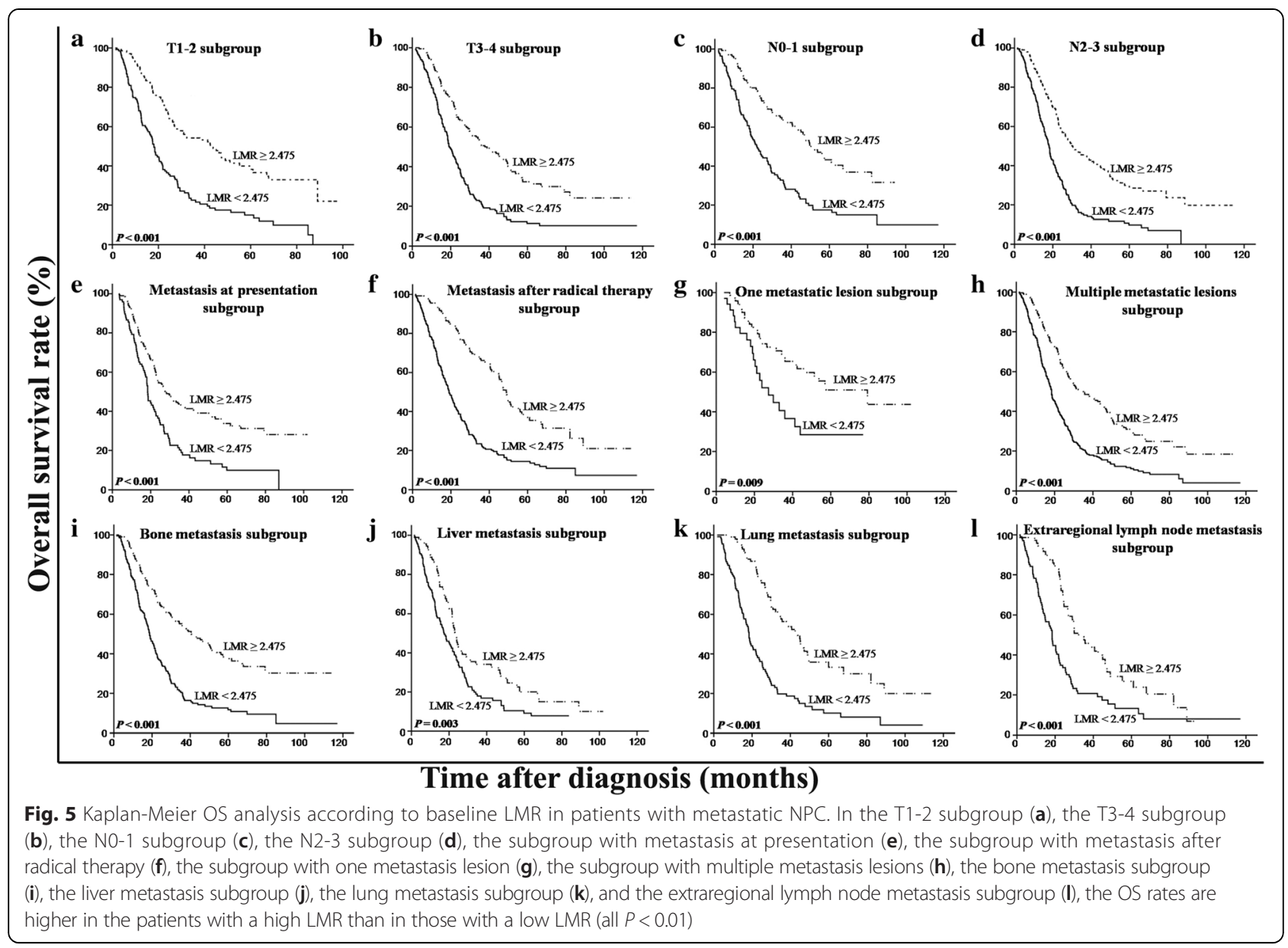

patients with metastatic NPC, LMR outperformed them in this regard. Our results of multivariate Cox proportional hazard analysis showed that LMR and absolute lymphocyte and monocyte counts were independent prognostic factors. However, after stratification analysis, only LMR remained a significant predictor of prognosis. In addition, consistent with the findings of Pan et al. [37], we found that an advanced $\mathrm{N}$ stage, the presence of two or more metastatic lesions, and liver metastasis were independent prognostic factors for short OS, whereas lung or bone metastases were not associated with OS. We postulate that these findings may be associated with a unique biological behavior of NPC in the liver metastasis group.

\section{Conclusion}

Our results show that LMR can function as an independent prognostic factor for patients with metastatic NPC. Moreover, this ratio can be easily determined with routine blood counts and is readily applicable clinically. We acknowledge that our findings are limited by the retrospective and single center nature of this study; thus, further independent validation of our findings is warranted.

\section{Competing interests}

The authors declare that they have no competing interests.

\section{Authors' contributions}

MYC conceived and designed the experiments; RJ, ZHY, XYC, YY, and XZ performed the experiments; RJ, LG, RS, DHL, QYC, and PYH analyzed the data; YQX, XL, LW, WXX, and HQM contributed reagents, materials, or analysis tools; RJ and MYC wrote the paper. All authors read and approved the final manuscript.

\section{Acknowledgments}

This study was supported by grants from Sun Yat-sen University Clinical Research 5010 Program (201310) and the National Natural Science Foundation of China (No. 81071890).

\section{Author details}

${ }^{1}$ Sun Yat-sen University Cancer Center; State Key Laboratory of Oncology in South China; Collaborative Innovation Center for Cancer Medicine, Guangzhou, Guangdong 510060, P. R. China. ²Department of Nasopharyngeal Carcinoma, Sun Yat-sen University Cancer Center, Guangzhou, Guangdong 510060, P. R. China. ${ }^{3}$ Department of Cancer Prevention, Guangzhou, Guangdong 510060, P. R. China. ${ }^{4}$ Department of 
Medical Oncology, Sun Yat-sen University Cancer Center, Guangzhou, Guangdong 510060, P. R. China. ${ }^{5}$ Department of Biochemistry, Zhongshan School of Medicine, Sun Yat-sen University, Guangzhou, Guangdong 510080, P. R. China.

\section{Received: 12 December 2014 Accepted: 9 April 2015}

Published online: 10 June 2015

\section{References}

1. Wei KR, Zheng RS, Zhang SW, Liang ZH, Ou ZX, Chen WQ. Nasopharyngeal carcinoma incidence and mortality in China in 2010. Chin J Cancer. 2014;33:381-7.

2. Adham M, Kurniawan AN, Muhtadi Al, Roezin A, Hermani B, Gondhowiardjo $S$, et al. Nasopharyngeal carcinoma in Indonesia: epidemiology, incidence, signs, and symptoms at presentation. Chin J Cancer. 2012;31:185-96.

3. Lee N, Xia P, Quivey JM, Sultanem K, Poon I, Akazawa C, et al. Intensitymodulated radiotherapy in the treatment of nasopharyngeal carcinoma: an update of the UCSF experience. Int J Radiat Oncol Biol Phys. 2002:53:12-22.

4. Lee AW, Sze WM, Au JS, Leung SF, Leung TW, Chua DT, et al. Treatment results for nasopharyngeal carcinoma in the modern era: the Hong Kong experience. Int J Radiat Oncol Biol Phys. 2005;61:1107-16.

5. Hui EP, Leung SF, Au JS, Zee B, Tung S, Chua D, et al. Lung metastasis alone in nasopharyngeal carcinoma: a relatively favorable prognostic group. A study by the Hong Kong Nasopharyngeal Carcinoma Study Group. Cancer. 2004;101:300-6.

6. Teo PM, Kwan WH, Lee WY, Leung SF, Johnson PJ. Prognosticators determining survival subsequent to distant metastasis from nasopharyngeal carcinoma. Cancer. 1996;77:2423-31.

7. Sun D, Elson P, Liedtke M, Medeiros BC, Earl M, Alizadeh A, et al. Absolute lymphocyte count at day 28 independently predicts event-free and overall survival in adults with newly diagnosed acute lymphoblastic leukemia. Am J Hematol. 2012:87:957-60.

8. Bruckner HW, Lavin PT, Plaxe SC, Storch JA, Livstone EM. Absolute granulocyte, lymphocyte, and monocyte counts. Useful determinants of prognosis for patients with metastatic cancer of the stomach. JAMA. 1982;247:1004-6.

9. He JR, Shen GP, Ren ZF, Qin H, Cui C, Zhang Y, et al. Pretreatment levels of peripheral neutrophils and lymphocytes as independent prognostic factors in patients with nasopharyngeal carcinoma. Head Neck. 2012;34:1769-76.

10. Schmidt H, Bastholt L, Geertsen P, Christensen IJ, Larsen S, Gehl J, et al. Elevated neutrophil and monocyte counts in peripheral blood are associated with poor survival in patients with metastatic melanoma: a prognostic model. Br J Cancer. 2005;93:273-8.

11. Koh YW, Kang HJ, Park C, Yoon DH, Kim S, Suh C, et al. The ratio of the absolute lymphocyte count to the absolute monocyte count is associated with prognosis in Hodgkin's lymphoma: correlation with tumor-associated macrophages. Oncologist. 2012;17:871-80.

12. Li J, Jiang R, Liu WS, Liu Q, Xu M, Feng QS, et al. A large cohort study reveals the association of elevated peripheral blood lymphocyte-tomonocyte ratio with favorable prognosis in nasopharyngeal carcinoma. PLoS One. 2013;8:e83069.

13. Zeng $Y$, Zhang LG, Wu YC, Huang YS, Huang NQ, Li JY, et al. Prospective studies on nasopharyngeal carcinoma in Epstein-Barr virus IgANCA antibody-positive persons in Wuzhou City. China Int J Cancer. 1985;36:545-7.

14. Xiao WW, Huang SM, Han F, Wu SX, Lu LX, Lin CG, et al. Local control, survival, and late toxicities of locally advanced nasopharyngeal carcinoma treated by simultaneous modulated accelerated radiotherapy combined with cisplatin concurrent chemotherapy: long-term results of a phase 2 study. Cancer. 2011;117:1874-83.

15. Chen MY, Jiang R, Guo L, Zou X, Liu Q, Sun R, et al. Locoregional radiotherapy in patients with distant metastases of nasopharyngeal carcinoma at diagnosis. Chin J Cancer. 2013:32:604-13.

16. Lai SZ, Li WF, Chen L, Luo W, Chen YY, Liu LZ, et al. How does intensitymodulated radiotherapy versus conventional two-dimensional radiotherapy influence the treatment results in nasopharyngeal carcinoma patients? Int J Radiat Oncol Biol Phys. 2011;80:661-8.

17. Roxburgh CS, McMillan DC. Role of systemic inflammatory response in predicting survival in patients with primary operable cancer. Future Oncol. 2010;6:149-63.
18. Teramukai S, Kitano T, Kishida Y, Kawahara M, Kubota K, Komuta K, et al. Pretreatment neutrophil count as an independent prognostic factor in advanced non-small-cell lung cancer: an analysis of Japan Multinational Trial Organisation LC00-03. Eur J Cancer. 2009;45:1950-8.

19. Kishi Y, Kopetz S, Chun YS, Palavecino M, Abdalla EK, Vauthey JN. Blood neutrophil-to-lymphocyte ratio predicts survival in patients with colorectal liver metastases treated with systemic chemotherapy. Ann Surg Oncol. 2009;16:614-22.

20. Porrata LF, Inwards DJ, Ansell SM, Micallef IN, Johnston PB, Hogan WJ, et al. Day 100 peripheral blood absolute Lymphocyte/Monocyte ratio and survival in classical Hodgkin's Lymphoma Postautologous peripheral blood Hematopoietic stem cell transplantation. Bone Marrow Res. 2013;2013:658371.

21. Li ZM, Huang JJ, Xia Y, Sun J, Huang Y, Wang Y, et al. Blood lymphocyte-tomonocyte ratio identifies high-risk patients in diffuse large B-cell lymphoma treated with R-CHOP. PLOS One. 2012;7:e41658.

22. Lin GN, Peng JW, Xiao JJ, Liu DY, Xia ZJ. Prognostic impact of circulating monocytes and lymphocyte-to-monocyte ratio on previously untreated metastatic non-small cell lung cancer patients receiving platinum-based doublet. Med Oncol. 2014;31:70.

23. Jin $Y$, Ye X, He C, Zhang B, Zhang Y. Pretreatment neutrophil-to-lymphocyte ratio as predictor of survival for patients with metastatic nasopharyngeal carcinoma. Head Neck. 2015;37:69-75.

24. Mantovani A, Allavena P, Sica A, Balkwill F. Cancer-related inflammation. Nature. 2008;454:436-44.

25. Coussens LM, Werb Z. Inflammation and cancer. Nature. 2002:420:860-7.

26. Moore MM, Chua W, Charles KA, Clarke SJ. Inflammation and cancer: causes and consequences. Clin Pharmacol Ther. 2010;87:504-8.

27. Rabinowich H, Cohen R, Bruderman I, Steiner Z, Klajman A. Functional analysis of mononuclear cells infiltrating into tumors: lysis of autologous human tumor cells by cultured infiltrating lymphocytes. Cancer Res. 1987:47:173-7.

28. Mohammed ZM, Going JJ, Edwards J, Elsberger B, Doughty JC, McMillan DC. The relationship between components of tumour inflammatory cel infiltrate and clinicopathological factors and survival in patients with primary operable invasive ductal breast cancer. Br J Cancer. 2012;107:864-73.

29. Cho H, Hur HW, Kim SW, Kim SH, Kim JH, Kim YT, et al. Pre-treatment neutrophi to lymphocyte ratio is elevated in epithelial ovarian cancer and predicts survival after treatment. Cancer Immunol Immunother. 2009;58:15-23.

30. Yamanaka T, Matsumoto S, Teramukai S, Ishiwata R, Nagai $Y$, Fukushima M. The baseline ratio of neutrophils to lymphocytes is associated with patient prognosis in advanced gastric cancer. Oncology. 2007;73:215-20.

31. Auffray C, Sieweke MH, Geissmann F. Blood monocytes: development, heterogeneity, and relationship with dendritic cells. Annu Rev Immunol. 2009:27:669-92.

32. Torisu-Itakura H, Lee JH, Huynh Y, Ye X, Essner R, Morton DL. Monocytederived IL-10 expression predicts prognosis of stage IV melanoma patients. J Immunother. 2007;30:831-8.

33. Anand M, Chodda SK, Parikh PM, Nadkarni JS. Abnormal levels of proinflammatory cytokines in serum and monocyte cultures from patients with chronic myeloid leukemia in different stages, and their role in prognosis. Hematol Oncol. 1998;16:143-54.

34. Pollard JW. Tumour-educated macrophages promote tumour progression and metastasis. Nat Rev Cancer. 2004:4:71-8.

35. Hefler L, Tempfer C, Heinze G, Mayerhofer K, Breitenecker G, Leodolter S, et al. Monocyte chemoattractant protein-1 serum levels in ovarian cancer patients. Br J Cancer. 1999;81:855-9.

36. Laughter AH, Twomey JJ. Suppression of lymphoproliferation by high concentrations of normal human mononuclear leukocytes. J Immunol. 1977:119:173-9.

37. Pan CC, Lu J, Yu JR, Chen P, Li W, Huang ZL, et al. Challenges in the modification of the M1 stage of the TNM staging system for nasopharyngeal carcinoma: A study of 1027 cases and review of the literature. Exp Ther Med. 2012:4:334-8. 